\title{
Review
}

nephron

Clinical

Practice

Published online: September 24, 2014

DOI: 10.1159/000363679

\section{Renal Recovery at Different Ages}

\author{
Stuart L. Goldstein \\ Center for Acute Care Nephrology, Cincinnati Children's Hospital Medical Center, University of \\ Cincinnati College of Medicine, Cincinnati, Ohio, USA
}

\section{Key Words}

Kidney disease progression - Pediatric patients · Renal recovery $\cdot$ Renal reserve

\section{Abstract}

While the field of acute kidney injury (AKI) research has undergone exponential growth over the past 15 years, the topic of renal recovery has only recently garnered much attention. Both lack of standardized renal recovery definitions and lack of systematic assessment of AKI survivors for chronic kidney disease development pose barriers to the complete understanding of the renal recovery epidemiology. In addition, evaluation of pediatric AKI renal recovery is further complicated by the potential AKI effects on renal development as well as a relatively greater renal reserve for younger children. The aims of this review are to review the current state of knowledge in pediatric AKI renal recovery.

\section{(c) 2014 S. Karger AG, Base}

All the world's a stage, And all the men and women merely players; They have their exits and their entrances, And one man in his time plays many parts,

His acts being seven ages. (As You Like It, Act II, Scene VII) William Shakespeare

\section{Introduction}

Acute kidney injury (AKI) complicates many pediatric and adult hospitalizations, with pediatric AKI rates ranging from 10 to $80 \%$ depending on the underlying severity of illness and comorbidities [1]. Pediatric AKI is consistently associated with increased morbidity in the critically ill child, with mortality rates reaching $50 \%$ in children receiving renal replacement therapy. As with adults, AKI provides an opportunity to prospectively follow survivors for the development of chronic kidney disease (CKD), yet, such follow-up has not been conducted systematically in either pediatric or adult populations [2]. In addition, a number of factors peculiar to the pediatric population pose barriers to accurate ascertainment of CKD in AKI survivors. The potential for renal recovery may be dependent on the stage of human development (perhaps not seven though), as the relationship between renal re-

Targeting Recovery from Acute Kidney Injury: Round Table Conference at the 19th International Conference on Continuous Renal Replacement Therapies (Manchester Grand Hyatt, San Diego, Calif., USA, March 2-3, 2014).

\section{KARGER}

E-Mail karger@karger.com www.karger.com/nec (c) 2014 S. Karger AG, Basel

$1660-2110 / 14 / 1274-0021 \$ 39.50 / 0$
Stuart L. Goldstein, MD

Cincinnati Children's Hospital Medical Cente

3333 Burnet Avenue, MLC 7022

Cincinnati, OH 45229 (USA)

E-Mail stuart.goldstein@cchmc.org 
serve and patient size and age can affect the detection of a functional decline. The aims of this article are to (1) review the current knowledge of recovery of kidney function after a pediatric AKI episode; (2) highlight the barriers to CKD ascertainment in pediatric AKI survivors, and (3) provide recommendations for a rational follow-up of children who survive an episode of AKI.

\section{Renal Recovery Definition}

The definition of renal recovery has not been standardized in the published AKI trials and is therefore multidimensional with both time and residual renal function components [3]. Pediatric studies have categorized renal recovery anywhere from dialysis independence during an intensive care unit or hospital stay, up to 3-5 years after the onset of the AKI episode. Such definitions are very similar to the range defined by various adult studies (e.g. the Acute Renal Failure Trial Network, ATN) or standardized classification systems [e.g. the Risk, Injury, Failure, Loss of Kidney Function, and End-Stage Renal Disease (RIFLE) criteria]. The recent Kidney Disease: Improving Global Outcomes (KDIGO) AKI work group developed a new entity of 'acute kidney disease' to bridge AKI to CKD, which serves as a first standard in this area [4].

Pediatric renal recovery ascertainment is challenged in a number of ways. First, no definition has been validated for neonatal AKI. Since preterm and term infant serum creatinine (and hence, kidney function assessment) reflects maternal creatinine, a creatinine-based AKI definition remains elusive due to the difficulty in determining the baseline creatinine. For example, a newborn's creatinine should decline from a maternal level of $0.8-1.2 \mathrm{mg} /$ $\mathrm{dl}$ to a normal level of $0.2-0.3 \mathrm{mg} / \mathrm{dl}$ in the1st week of life. Failure of the creatinine to decrease in that time frame or to that level could potentially be viewed as AKI. The recent NIH-sponsored Neonatal Acute Kidney Injury Workshop (http://www.niddk.nih.gov/news/eventscalendar/Pages/neonatal-acute-kidney-injury-workshop.aspx) will yield a consensus neonatal AKI definition that must be tested.

Infant glomerular filtration rate (GFR) increases over the 1st year of life from 15 to $90 \mathrm{ml} / \mathrm{min} / 1.73 \mathrm{~m}^{2}$, resulting in difficulty in deriving an AKI threshold [5]. The current CKD studies and definitions exclude children under 1 year of age; thus, the diagnosis of CKD cannot be made in small children at this point [6]. Finally, children are born with substantial relative renal reserve, with more than sufficient clearance capacity in relation to their met- abolic output. Thus, even if an AKI episode leads to significant kidney damage, functional impairment manifested by change in serum creatinine-based estimated GFR (eGFR) may not become manifest until a child enters their adolescent growth spurt with the associated increase in metabolic load.

\section{Recovery at Different Ages}

\section{Neonatal Renal Recovery}

In addition to the difficulties noted above, a number of issues unique to the neonatal population pose challenges to AKI to CKD ascertainment. First, since glomerulogenesis is not complete until 36 weeks of gestation, an AKI episode in preterm infants could theoretically cause irreversible developmental injury to the nephron. Should such an injury be classified as CKD, especially in light of the fact that the rate of CKD itself, independent of AKI, is 2-fold higher in preterm versus term infants [7]? No national guidelines exist for systematic serum creatinine measurement either during the neonatal course or in long-term survivor cohorts. Thus, AKI and CKD ascertainment is driven by the local standard of clinical care.

Very few studies have looked at AKI in neonates, and only two studies have reported on long-term follow-ups of AKI in this population. Abitbol et al. [8] assessed chronic kidney injury in 20 extremely low-birth-weight $(<1,000 \mathrm{~g})$ infants at least 3 years after an AKI episode. AKI was defined as a serum creatinine $>2 \mathrm{mg} / \mathrm{dl}$ for more than $48 \mathrm{~h}$ or oliguria $<0.5 \mathrm{ml} / \mathrm{kg} / \mathrm{h}$ for $24 \mathrm{~h}$. Infants with decreased GFR after $>3$ years of follow-up were noted to have had higher urinary protein excretion and serum creatinine at 1 year of age. These data argue for serum creatinine and urine protein screening at 1 year of age in premature infants with AKI. We recently reported pilot data from 51 survivors of cardiopulmonary bypass surgery 5-8 years after enrollment in a study of urinary AKI biomarkers [9]. While no difference in GFR, urine protein or hypertension rates were seen between the AKI+ $(\mathrm{n}=33)$ and AKI- $(\mathrm{n}=18)$ survivors, urinary kidney injury molecule 1 , interleukin-18 and liver-type fatty acid binding protein concentrations were all higher in AKI+ patients 5-8 years after AKI. These data suggest that damage may persist in AKI+ patients and that a functional change may not be apparent for years to come.

\section{Pediatric Renal Recovery at Hospital Discharge}

Hui-Stickle et al. [10] performed the largest contemporary assessment $(n=254$ children $)$ of pediatric AKI in
Goldstein 
a single center from 1999 to 2001. In their study, AKI was defined by hospital discharge or death summary and verified with a measured eGFR of $<75 \mathrm{ml} / \mathrm{min} / 1.73 \mathrm{~m}^{2}$. One hundred seventy-six patients survived to hospital discharge, with $66 \%$ recovering back to a baseline serum creatinine, $29 \%$ with incomplete recovery, and $5 \%$ requiring dialysis at the time of discharge. Ball and Kara [11] found that $40 \%$ of AKI survivors had decreased GFR, proteinuria, or hypertension at the time of hospital discharge in New Zealand. Thus, one third to one fourth of pediatric AKI survivors likely warrant long-term follow-up for CKD development.

\section{Pediatric Renal Recovery after Hospital Discharge}

Askenazi et al. [12] assessed long-term kidney injury and function 3-5 years after AKI in the patients from the Hui-Stickle cohort. While 11 patients were dialysis dependent at the time of discharge, another 5 patients developed end-stage renal disease during the follow-up period. Seventeen of 29 patients who returned for assessment had at least one sign of $\mathrm{CKD} /$ chronic kidney injury (low GFR, hyperfiltration, proteinuria, or hypertension), and more than half of these had two or more signs.

Mammen et al. [13] assessed renal recovery in 126 of 299 children for a minimum of 1 year after an AKI episode in the intensive care unit. In their study, CKD was defined as an eGFR $<60 \mathrm{ml} / \mathrm{min} / 1.73 \mathrm{~m}^{2}$, 'CKD risk' was defined as an eGFR of $60-90$ or $>150 \mathrm{ml} / \mathrm{min} / 1.73 \mathrm{~m}^{2}$. Abnormal GFR was observed in $57.1 \%$ of patients (CKD in $10.3 \%$ and $46.8 \%$ were at risk of CKD). These two studies, albeit small in size, demonstrate that CKD is a common occurrence in pediatric AKI survivors. While the actual percentages may be inflated due to positive ascertainment bias due to the incomplete assessment of the total cohorts, there were no demographic differences noted in either study between patients who participated and those who did not.

Garg et al. [14] performed a systematic review of 49 studies, comprising 3,476 patients who had hemolytic uremic syndrome, which is predominantly a pediatric disease. The pooled average of decreased GFR $(<80 \mathrm{ml} /$ $\min / 1.73 \mathrm{~m}^{2}$ ) was $14.8 \%$ (12\% died or had end-stage renal disease at the time of follow-up). Risk factors for CKD included prolonged oliguria or the need for dialysis for $>4$ weeks.

\section{Renal Recovery in Young Adult and Older Patients}

Renal recovery in older patients will be covered extensively in the remainder of this edition. However, it is important to note that most $\mathrm{AKI}$ and renal recovery studies in the 'adult' age group actually have assessed patients $>60$ years of age, even though the methods of adult studies always state that patients $>18$ years of age are eligible for enrollment. In fact, two of the largest adult AKI studies conducted in the last 5 years, the ATN and the Randomized Evaluation of Normal versus Augmented Levels of Renal Replacement Therapy (RENAL) trials $[15,16]$, only had a combined 54 patients (total: 2,589 patients, $2.1 \%$ ) aged 18-25 years enrolled [Paul Palevsky (Principal Investigator, ATN) and Rinaldo Bellomo (Principal Investigator, RENAL), pers. commun.]. Thus, we have little published data describing AKI rates in young adults to early middle-aged individuals, and, as a result, little knowledge of their renal recovery rates. Finally, as noted in other reviews in this volume, renal recovery from AKI in older individuals may be difficult to disentangle from the natural progression of loss of renal reserve and CKD development in the absence of AKI. In this way, the elderly are akin to the neonate who may lose renal reserve from AKI due to an impact on nephron development.

\section{Conclusions}

As was true 10 years ago before the development and validation of novel, multidimensional AKI classification systems, we are living in a similar definition confusion state with respect to renal recovery. Renal recovery ascertainment may be further confounded by the natural history of nephron development, renal reserve, and nephron loss at ends of the human age continuum. Progress in the field of renal recovery will depend on standardized definitions of functional recovery, and, perhaps, validation of novel damage biomarkers to yield early clues with respect to the progression of AKI to CKD.

References

1 Fortenberry JD, Paden ML, Goldstein SL: Acute kidney injury in children: an update on diagnosis and treatment. Pediatr Clin North Am 2013;60:669-688.

$\checkmark 2$ Goldstein SL, Jaber BL, Faubel S, Chawla LS Acute Kidney Injury Advisory Group of American Society of Nephrology: AKI transition of care: a potential opportunity to detect and prevent CKD. Clin J Am Soc Nephrol 2013;8:476-483.

-3 Goldstein SL, Chawla L, Ronco C, Kellum JA Renal recovery. Crit Care 2014;18:301.

4 Kidney Disease: Improving Global Outcomes (KDIGO) Acute Kidney Injury Work Group: KDIGO Clinical Practice Guideline for Acute Kidney Injury. Kidney Int Suppl 2012;2:1138. 
5 Askenazi DJ, Ambalavanan N, Goldstein SL: Acute kidney injury in critically ill newborns: what do we know? What do we need to learn? Pediatr Nephrol 2009;24:265-274.

-6 Schwartz GJ, Munoz A, Schneider MF, Mak RH, Kaskel F, Warady BA, Furth SL: New equations to estimate GFR in children with CKD. J Am Soc Nephrol 2009;20:629-637.

7 White SL, Perkovic V, Cass A, Chang CL, Poulter NR, Spector T, Haysom L, Craig JC, Salmi IA, Chadban SJ, Huxley RR: Is low birth weight an antecedent of CKD in later life? A systematic review of observational studies. Am J Kidney Dis 2009;54:248-261.

8 Abitbol CL, Bauer CR, Montane B, Chandar J, Duara S, Zilleruelo G: Long-term follow-up of extremely low birth weight infants with neonatal renal failure. Pediatr Nephrol 2003; 18:887-893.
9 Claes DJ, Cooper DS, Menon S, Bennett M, Ma Q, Krawczeski C, Goldstein SL: Novel urinary biomarkers remain elevated 5-8 y after AKI due to cardiac surgery in children as compared to controls (abstract). Pediatr Res 2013.

10 Hui-Stickle S, Brewer ED, Goldstein SL: Pediatric ARF epidemiology at a tertiary care center from 1999 to 2001. Am J Kidney Dis 2005; 45:96-101.

11 Ball EF, Kara T: Epidemiology and outcome of acute kidney injury in New Zealand children. J Paediatr Child Health 2008;44:642646.

12 Askenazi DJ, Feig DI, Graham NM, HuiStickle S, Goldstein SL: 3-5 year longitudinal follow-up of pediatric patients after acute renal failure. Kidney Int 2006;69:184-189.

13 Mammen C, Al Abbas A, Skippen P, Nadel H, Levine D, Collet JP, Matsell DG: Long-term risk of CKD in children surviving episodes of acute kidney injury in the intensive care unit: a prospective cohort study. Am J Kidney Dis 2012;59:523-530.
4 Garg AX, Suri RS, Barrowman N, Rehman F, Matsell D, Rosas-Arellano MP, Salvadori M, Haynes RB, Clark WF: Long-term renal prognosis of diarrhea-associated hemolytic uremic syndrome: a systematic review, metaanalysis, and meta-regression. JAMA 2003; 290:1360-1370.

15 Palevsky PM, Zhang JH, O'Connor TZ, Chertow GM, Crowley ST, Choudhury D, Finkel K, Kellum JA, Paganini E, Schein RM, Smith MW, Swanson KM, Thompson BT, Vijayan A, Watnick S, Star RA, Peduzzi P: Intensity of renal support in critically ill patients with acute kidney injury. N Engl J Med 2008;359: 7-20.

16 Bellomo R, Cass A, Cole L, Finfer S, Gallagher M, Lo S, McArthur C, McGuinness S, Myburgh J, Norton R, Scheinkestel C, Su S: Intensity of continuous renal-replacement therapy in critically ill patients. N Engl J Med 2009;361:1627-1638. 